\title{
Anastrozole-induced Autoimmune Hepatitis: A Rare Complication of Breast Cancer Therapy
}

\author{
OLEKSANDRA KLAPKO $^{1}$, ELIE GHOULAM ${ }^{2}$, SHRIRAM JAKATE ${ }^{3}$, SHEILA ESWARAN $^{2}$ and LYDIA USHA ${ }^{1}$ \\ ${ }^{1}$ Division of Hematology/Oncology and Stem Cell Transplant, and ${ }^{2}$ Section of Hepatology, Department of Medicine, \\ and ${ }^{3}$ Department of Pathology, Rush University Medical Center, Chicago, IL, U.S.A.
}

\begin{abstract}
Background: Autoimmune hepatitis (AIH) is an extremely rare complication of anastrozole therapy. It presents with elevated liver function tests. The diagnosis is established by detecting high titers of autoantibodies such as antinuclear antibodies, anti-smooth muscle antibodies, and elevated immunoglobulins. It is confirmed with a liver biopsy showing interface rosetting and an increased number of plasma cells. Early diagnosis of anastrozole-induced AIH is important because it allows anastrozole to be discontinued and immunomodulatory treatment to be promptly initiated. Case Report: We present the case of a 71-year-old female patient diagnosed with early-stage breast cancer. The patient developed AIH as a result of treatment with anastrozole. Its clinicopathological presentation, diagnosis, and treatment are reviewed. Conclusion: This case report intends to make clinicians aware of this rare complication of anastrozole therapy. AIH should be suspected in any patient on anastrozole (and possibly, other aromatase inhibitors) who develops elevated liver function tests.
\end{abstract}

Anastrozole is a third-generation non-steroidal aromatase inhibitor (Figure 1) commonly utilized in the adjuvant and metastatic setting to treat patients with postmenopausal estrogen receptor-positive breast cancer. Aromatase inhibitors (AIs) suppress plasma estrogen levels by inhibiting or inactivating aromatase, the enzyme responsible for the peripheral conversion of androgens to estrogens (1). Anastrozole is usually well tolerated, but it can occasionally cause side-effects (commonly, hot flashes, arthralgia, decreased bone density (2) that are usually mild

Correspondence to: Lydia Usha, MD, Department of Hematology and Oncology, Rush University Medical Center, 1725 W. Harrison St., Ste 809, Chicago, IL 60612, U.S.A. Tel: +1 3125632200, Fax: +1 3129423192, e-mail: lydia_usha@rush.edu

Key Words: Anastrozole, adverse effects, autoimmune hepatitis, drug-induced liver injury, breast cancer.
Autoimmune hepatitis (AIH) as a result of anastrozole therapy is very uncommon $(3,4)$. Here we report the development of AIH in a patient receiving anastrozole for adjuvant treatment of early-stage breast cancer.

\section{Case Report}

A 71-year-old female was diagnosed with screening-detected early-stage breast cancer in July, 2013. A diagnostic mammogram showed an area of irregular density associated with microcalcifications in the upper outer left breast. A left breast biopsy revealed an infiltrating ductal carcinoma with tubular features which was $0.3-\mathrm{cm}$ long, grade 1 , estrogenpositive, progesterone-positive, and human epidermal growth factor receptor $2 /$ neu-negative.

The patient's past medical history included hypertension, unspecified hypothyroidism, dry-eye syndrome and migraines. She had no prior history of liver disease, no history of alcohol abuse or exposure to hepatotoxins, and no family history of liver disease. Her medications included ibuprofen, acetaminophen, levothyroxine, methyclothiazide, potassium chloride, cyclosporine eye drops and multivitamins.

The patient underwent left lumpectomy and sentinel lymph node biopsy, which showed no residual invasive cancer. The pathological stage was T1aN0 (clinical stage I). The patient subsequently underwent a course of hypofractionated adjuvant radiation (completed in November, 2013) and was started on adjuvant $1 \mathrm{mg}$ anastrozole daily in December, 2013.

In September 2014, on routine blood work (Table I), the patient was found to have elevated liver function tests (LFTs). A re-staging computed tomographic scan with contrast in October 2014 was negative for liver metastases; however, it showed hypodense liver parenchyma relative to the spleen, which was consistent with hepatic steatosis. The decision was made to monitor the patient's LFTs, which continued to rise in February, 2015. At that time, the patient was referred to hepatology for further work-up.

A hepatology work-up in March 2015 revealed antinuclear antibodies (ANA) with high titer $(1: 1,280)$ and elevated 
Table I. The patient's laboratory results (abnormal values are shown in bold).

\begin{tabular}{|c|c|c|c|c|c|c|c|c|c|c|}
\hline Date of testing & $\begin{array}{l}\text { AST } \\
(\mathrm{U} / \mathrm{l})\end{array}$ & $\begin{array}{l}\text { ALT } \\
(\mathrm{U} / 1)\end{array}$ & $\begin{array}{l}\text { Bilirubin total } \\
(\mathrm{mg} / \mathrm{dl})\end{array}$ & $\begin{array}{l}\text { ALP } \\
(\mathrm{U} / \mathrm{l})\end{array}$ & $\begin{array}{l}\text { ANA } \\
\text { titer }\end{array}$ & $\begin{array}{c}\text { SSA } \\
\text { antibody }\end{array}$ & $\begin{array}{l}\text { Platelets } \\
\left(10^{3} / \mu \mathrm{l}\right)\end{array}$ & INR & $\begin{array}{l}\text { Creatinine } \\
(\mathrm{mg} / \mathrm{dl})\end{array}$ & $\begin{array}{l}\text { Albumin } \\
(\mathrm{g} / \mathrm{dl})\end{array}$ \\
\hline Normal value & $3-44$ & $0-40$ & $0.2-1.3$ & $30-125$ & $<1: 40$ & $0.00-0.89$ & $150-399$ & $0.8-1.2$ & $0.65-1.00$ & $3.5-5.0$ \\
\hline $8 / 6 / 2013$ & 35 & 39 & 0.9 & 93 & & & 304 & 0.98 & 0.74 & 3.9 \\
\hline $9 / 9 / 2014$ & 109 & 130 & 0.9 & 95 & & & & & & 3.5 \\
\hline $10 / 10 / 2014$ & 91 & 106 & 0.9 & 98 & & & 279 & 1.0 & 0.98 & 3.7 \\
\hline $2 / 23 / 2015$ & 126 & 115 & 1.0 & 106 & & & 307 & & 0.92 & 3.8 \\
\hline $3 / 5 / 2015$ & & & & & 1:1280 & $>3.5$ & & & & \\
\hline $3 / 24 / 2015$ & 75 & 92 & 1.0 & 102 & & & & 0.99 & 0.94 & 3.9 \\
\hline $4 / 16 / 2015$ & 46 & 61 & 1.3 & 83 & & & 297 & & & \\
\hline $4 / 23 / 2015$ & 26 & 34 & 0.7 & 87 & & & 287 & & 1.02 & 4.4 \\
\hline $6 / 26 / 2015$ & 64 & 39 & 1.0 & 72 & & & & & 1.0 & 4.6 \\
\hline $9 / 23 / 2015$ & 21 & 15 & 0.7 & 74 & & & 229 & & 0.97 & 4.3 \\
\hline $12 / 21 / 2015$ & & & & & 1:1280 & $>3.5$ & & & & \\
\hline $1 / 15 / 2016$ & 21 & 12 & 0.7 & 90 & & & 299 & & 0.89 & 4.0 \\
\hline $8 / 1 / 2016$ & 20 & 11 & 0.8 & 58 & & & & & & 4.4 \\
\hline
\end{tabular}

AST, Aspartate aminotransferase; ALT, alanine transaminase; ALP, alkaline phosphatase; ANA, antinuclear antibody; SSA antibody, anti-Sjögren'ssyndrome-related antigen A antibody; INR, international normalized ratio.

antibodies to Sjögren's-syndrome-related antigen A (SSA). Antibodies to smooth muscle (ASMA) and anti-mitochondrial antibodies (AMA) were negative. Serum immunoglobulins were normal. Hepatitis A, B and C were ruled out. Since the results of serological and imaging tests were inconclusive, a liver biopsy was performed in March 2015. A pathological examination showed grade 2 chronic hepatitis with an increased number of plasma cells at the portal-lobular interface and focal rosetting characteristic of AIH (Figure 2). Based on these findings, the diagnosis of anastrozole-induced AIH was made. In May 2015, it was recommended that the patient discontinue anastrozole and start treatment with prednisone and azathioprine. She completed a prednisone taper over 3 months and continued azathioprine. The patient's LFTs improved with the removal of anastrozole and the initiation of immunomodulatory therapy with steroids and azathioprine. This favorable response confirmed the diagnosis of anastrozole-induced AIH.

The patient has been off anastrozole since March 2015. The last LFTs in March 2017 were normal. As of this writing, the plan is to discontinue azathioprine 18 months after normalization of LFTs and continue monitoring the patient. She was diagnosed with cutaneous lupus erythematosus by punch biopsy of the right upper extremity in December 2015, however, she remains breast cancer-free.

\section{Discussion}

Anti-hormonal therapy is recommended for adjuvant therapy of women with early-stage hormone-receptor (estrogen/ progesterone receptor)-positive breast cancer with tumor size of $0.5 \mathrm{~cm}$ or larger and considered in women with tumor size of $0.5 \mathrm{~cm}$ or smaller (5).

In postmenopausal women, AIs (anastrozole, letrozole, and exemestane) are most frequently used as they have increased efficacy and a more favorable side-effect profile compared to tamoxifen $(6,7)$. An AI is taken daily for at least 5 years. Benefits include a $50 \%$ or more decrease in the risk of distant and contralateral breast cancer recurrence, and a small decrease in the risk of ipsilateral breast cancer (8). Anastrozole is the most frequently prescribed AI.

Some of the most common adverse effects of anastrozole (ones that occur in more than $10 \%$ of patients) are hot flashes, arthralgia, osteoporosis, fractures, depression, nausea and vomiting. Hepatotoxicity is relatively rare $(<10 \%$ of patients) (2). It usually presents with elevated liver function tests in serum (alkaline phosphatase, aspartate aminotransferase and alanine transaminase), which occur in $2 \%$ to $5 \%$ of patients on anastrozole. Another form of hepatotoxicity is hepatic steatosis, which has been reported to occur in up to $30 \%$ of long-term anastrozole users (9).

With an extensive literature review, we found a description of only two anastrozole-induced AIH cases (3, 4). Interestingly, both cases involved older women (66 and 70 years old). One possible explanation for the ability of anastrozole to cause $\mathrm{AIH}$ is its ability to affect the immune system via impaired self-tolerance (3).

Medications notorious for causing AIH include amoxicillinclavulanate, minocycline, statins, and diclofenac. Herbal supplements such as black cohosh can cause AIH as well (10). In oncology, immunotherapeutic agents such as ipilimumab, a monoclonal antibody to cytotoxic T-lymphocyte antigen 4, 


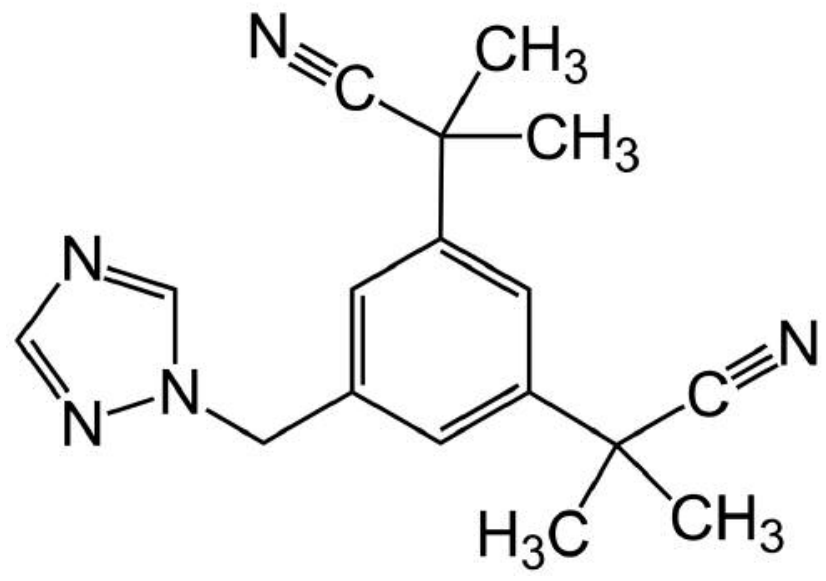

Figure 1. Chemical formula of anastrozole.

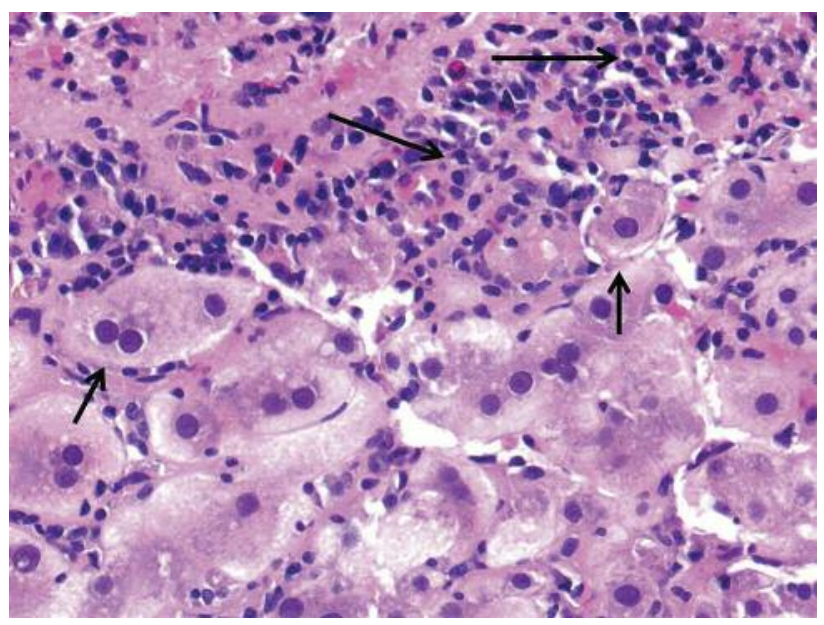

Figure 2. Microphotograph of the liver biopsy showing increased numbers of plasma cells at the portal-lobular interface (long arrows) and rosetting (short arrows) at the periphery of the lobules (hematoxylin and eosin stain; $\times 400$ magnification).

have been shown to induce AIH (11). Female gender and genetic predisposition to autoimmune diseases are considered risk factors for development of drug-induced AIH (12). We believe that our patient had a predisposition to autoimmune diseases given her history of hypothyroidism, dry eyes, AIH, and finally, development of cutaneous lupus.

It is important for clinicians to be vigilant about the possibility of drug-induced liver injury, including druginduced AIH. The presentation of our patient is characteristic of drug-induced AIH: i) the temporal relationship of elevated LFTs following the initiation of anastrozole; ii) the pattern of elevated aminotransferases with normal or marginally elevated alkaline phosphatase and bilirubin; iii) elevated titers of autoantibodies, including ANA; iv) extrahepatic autoimmune manifestations; v) favorable response to immunosuppressive therapy (12).

A liver biopsy is utilized to confirm the clinical diagnosis in such cases. The typical pathological manifestations of $\mathrm{AIH}$ include chronic hepatitis with increased numbers of plasma cells at the interface between lobules and portal tracts of the liver. In addition, the portal-lobular interface typically shows rosettes, which are ball-like structures composed of isolated hepatocytes or small groups of hepatocytes surrounded by linearly arranged lymphocytes and plasma cells. Depending upon the duration and severity of $\mathrm{AIH}$, hepatitis can be associated with fibrosis.

AIH is an inflammation of the liver of unknown etiology. The pathogenesis is likely multifactorial: environmental triggers, a failure of immune tolerance mechanisms, and a genetic predisposition to induce a T-cell-mediated immune attack upon liver antigens, leading to a progressive necroinflammatory and fibrotic process in the liver (13). The prognosis of AIH depends on the presence of cirrhosis at the time of diagnosis and on the response to immunomodulatory therapy (14).

In this case, a liver biopsy was crucial in order to exclude other causes of liver disease, including anastrozolerelated steatosis. Although steatosis was described on computed tomographic imaging, our patient did not have steatosis on biopsy.

It is often challenging to differentiate between idiopathic spontaneous AIH, drug-induced $\mathrm{AIH}$ and other types of drug-induced liver injury. However, in our patient, the timing of onset after initiation of anastrozole, positivity for ANA, the pattern of LFTs elevation and their improvement with the discontinuation of anastrozole, and the response to beginning immunosuppressive therapy, point towards druginduced AIH (10).

\section{Conclusion}

AIH is an extremely rare complication of anastrozole therapy. However, clinicians must be aware of it so as to recognize it as a potential cause of elevated liver enzymes in a patient recently started on an AI. Once AIH is suspected, a clinician should order a blood test for ANA, ASMA and quantitative immunoglobulins, and refer the case to a hepatologist. Elevated titers of these antibodies establish the diagnosis. In less clear cases, a liver biopsy may be necessary to confirm the diagnosis. It is important to diagnose this condition early, to promptly discontinue anastrozole, and to start immunomodulatory therapy as soon as possible. These measures can prevent liver cirrhosis and fatal hepatic failure. This case also underscores the 
importance of carefully weighing the risks and benefits of aromatase inhibitors in the adjuvant setting, especially in elderly women with low-risk, early-stage breast cancer.

\section{References}

1 Buzdar A and Howell A: Advances in aromatase inhibition: Clinical efficacy and tolerability in the treatment of breast cancer. Clin Cancer Res 7(9): 2620-2635, 2001.

2 Arimidex [package insert]. AstraZeneca Pharmaceuticals LP, Wilmington, DE; 2009. Available at: http://www. accessdata.fda.gov/drugsatfda_docs/label/2011/020541s026lbl.pd f. [Accessed date 6 December, 2016].

3 Inno A, Basso M, Vecchio FM, Marsico VA, Cerchiaro E, D'Argento E, Bagala C and Barone C: Anastrozole-related acute hepatitis with autoimmune features: a case report. BMC Gastroenterol 11: 32, 2011.

4 Islam MS, Wright G, Tanner P and Lucas R: A case of anastrozole-related drug-induced autoimmune hepatitis. Clinical J Gastroenterol 7(5): 414-417, 2014.

5 NCCN Clinical Practice Guidelines in Oncology (NCCN Guidelines ${ }^{\circledR}$ ) for Breast Cancer: Version 2.2016. (C) National Comprehensive Cancer Network, Inc 2017. Available at: https://www.nccn.org/professionals/physician_gls/f_guidelines.as p. [Accessed date 2 January, 2017].

6 Smith IE and Dowsett M: Aromatase inhibitors in breast cancer. N Engl J Med 348(24): 2431-2442, 2003.

7 Buzdar AU and Cuzick J: Anastrozole as an adjuvant endocrine treatment for postmenopausal patients with breast cancer: emerging data. Clin Cancer Res 12(3 suppl): S1037-1048, 2006.

8 Dowsett M, Forbes JF, Bradley R, Ingle J, Aihara T, Bliss J, Boccardo F, Coates A, Coombes RC, Cuzick J, Dubsky P, Gnant M, Kaufmann M, Kilburn L, Perrone F, Rea D, Thurlimann B, van de Velde C, Pan H, Peto R, Davies C and Gray R; Early Breast Cancer Trialists' Collaborative Group: Aromatase inhibitors versus tamoxifen in early breast cancer: patient-level meta-analysis of the randomised trials. Lancet 386(10001): 1341-1352, 2015.
9 Lin Y, Liu J, Zhang X, Li L, Hu R, Liu J, Deng Y, Chen D, Zhao Y, Sun S, Ma R, Zhao Y, Liu J, Zhang Y, Wang X, Li Y, He P, Li E, Xu Z, Wu Y, Tong Z, Wang X, Huang T, Liang Z, Wang S, Su F, Lu Y, Zhang H, Feng G and Wang S: A prospective, randomized study on hepatotoxicity of anastrozole compared with tamoxifen in women with breast cancer. Cancer Science 105(9): 1182-1188, 2014.

10 Licata A, Maida M, Cabibi D, Butera G, Macaluso FS, Alessi N, Caruso C, Craxi A and Almasio PL: Clinical features and outcomes of patients with drug-induced autoimmune hepatitis: a retrospective cohort study. Dig Liver Dis 46(12): 1116-1120, 2014.

11 Ravi S, Spencer K, Ruisi M, Ibrahim N, Luke JJ, Thompson JA, Shirai K, Lawson D, Bartell H, Kudchadkar R, Gunter NT, Mehnert JM and Lipson EJ: Ipilimumab administration for advanced melanoma in patients with pre-existing hepatitis B or C infection: a multicenter, retrospective case series. J Immunother Cancer 2(1): 33, 2014.

12 Castiella A, Zapata E, Lucena MI and Andrade RJ: Druginduced autoimmune liver disease: A diagnostic dilemma of an increasingly reported disease. World J Hepatol 6(4): 160-168, 2014.

13 Manns MP, Czaja AJ, Gorham JD, Krawitt EL, Mieli-Vergani G, Vergani D and Vierling JM: Diagnosis and management of autoimmune hepatitis. Hepatology 51(6): 2193-2213, 2010.

14 Feld JJ, Dinh H, Arenovich T, Marcus VA, Wanless IR and Heathcote EJ: Autoimmune hepatitis: effect of symptoms and cirrhosis on natural history and outcome. Hepatology 42(1): 53$62,2005$. 